\title{
A high rate of modestly elevated plasma normetanephrine in a population referred for suspected PPGL when measured in a seated position
}

\author{
Jessica Boyd ${ }^{1,2}$, Alexander A Leung ${ }^{3}$, Hossein SM Sadrzadeh ${ }^{1,2}$, Christina Pamporaki ${ }^{4}$, Karel Pacak ${ }^{5}$, \\ Timo Deutschbein ${ }^{6}$, Stephanie Fliedner ${ }^{7}$ and Gregory A Kline ${ }^{3}$
}

${ }^{1}$ Department of Clinical Pathology, Faculty of Medicine, University of Calgary, Calgary, Alberta, Canada, ${ }^{2}$ Alberta Public Laboratory, Calgary, Alberta, Canada, ${ }^{3}$ Department of Medicine/Endocrinology, Cumming School of Medicine, University of Calgary, Calgary, Alberta, Canada, ${ }^{4}$ Department of Endocrinology, University Hospital, Carl Gustav Carus at TU Dresden, Dresden, Germany, ${ }^{5}$ Section of Medical Neuroendocrinology, Eunice Kennedy Shriver National Institute of Child Health and Human Development, National Institutes of Health, Bethesda, Maryland, USA, ${ }^{6}$ Division of Endocrinology and Diabetes, Department of Internal Medicine I, University Hospital Wuerzburg, University of Wuerzburg, Wuerzburg, Germany, and ${ }^{7}$ Neuroendocrine Oncology and Metabolism, First Department of Medicine, University Medical Center SchleswigHolstein, Lübeck, Germany

Correspondence should be addressed to G A Kline Email Gregory.kline@ahs.ca

\begin{abstract}
Objective: Determine rate of high plasma normetanephrine or metanephrine (PNM-PMN) in a large sample of patients according to PNM-PMN posture and age-adjusted references.

Design: Retrospective re-analysis of PNM-PMN from a Canadian reference laboratory ( $n=5452), 2011-2015$; most were in seated position $(n=5112)$ rather than supine $(n=340)$. An international PPGL database demonstrated expected distribution of supine PNM-PMN in PPGL patients.

Methods: All PNM-PMN from a tertiary referral laboratory were reviewed. Any PNM-PMN result greater than $2 \times$ upper reference limit (URL) was considered likely true PPGL. Results 1-2x URL were uncertain, requiring additional testing/follow-up despite most being false positive given the rarity of PPGL. The rate of results in the 1-2x URL category were calculated for each group according to collection posture and differing published URL: seated, supine or supine age adjusted.

Results: When collected and interpreted by seated URL, $19.6 \%$ of PNM required additional testing; only $4.6 \%$ being $>2 \times$ URL. For patients over age 50 years, the abnormal rate was $24.9 \%$. When collected supine, interpreted by supine age-adjusted URL, only 5.3\% of PNM were mildly elevated. Possible false positives may be even lower when considering PMN or plasma methoxytyramine which were commonly high in true PPGL despite mild PNM elevations. Conclusions: In a general medical population, seated PNM has a high rate of abnormal results, far exceeding expected prevalence. Supine measurement with supine, age-adjusted interpretation is strongly preferred prior to costly or invasive PPGL investigations.

Summary: Review of 5452 plasma normetanephrine measurements showed $20 \%$ to be high, likely false positives for most. Supine, age-adjusted measures were half as likely to be elevated.
\end{abstract}




\section{Introduction}

Pheochromocytoma and paraganglioma (PPGL) are rare endocrine tumors that may secrete catecholamines causing hypertension and other varied clinical features (1). Although most non-familial PPGL are benign, malignant forms are more common than previously thought (2). Despite the relative rarity of PPGLs in the general population, most physicians are aware of the dangers of these tumors and their potential to cause severe hypertension, arrhythmia or even death (3). Indications for PPGL screening often include patients with resistant or paroxysmal hypertension, known adrenal masses, syndromic presentations pointing toward familial PPGL and surveillance for recurrent PPGL disease after surgery. However, PPGL screening is commonly undertaken as part of the investigation into episodic 'spells' (e.g. hypertension, sweating, palpitations presenting in a patient without any obvious trigger) seen in both primary care and specialist clinics. Given the very diverse and often undifferentiated presentations leading to PPGL screening, clinical laboratories may receive a high volume of referrals for testing for what is still a rare disease.

The most commonly recommended biochemical screening tests are the urine or plasma determination of fractionated normetanephrine and metanephrine (4). Although the latest guidelines recommend either test may be used first line, there is now evidence to suggest that measurements of plasma free normetanephrine (PNM) and metanephrine (PMN) provide significantly better performance over urinary NM-MN in terms of sensitivity and specificity, when performed according to strictly controlled posture and collection conditions $(5,6,7)$. In the years since PNM-PMN has become more widely available, there has been a marked increase in general utilization of this test even outside of specialty endocrine centers.

A concern arises however when the PNM-PMN test is performed in less selected populations and with less strict attention to posture or patient preparation. The sensitivity, specificity and positive predictive value of supine, resting PNM-PMN has been repeatedly published $(8,9,10)$ along with a warning about the marked loss of specificity when PNM-PMN are measured in a seated position and/or not interpreted according to age-specific normal ranges $(11,12,13)$. Many clinical laboratories lack the facilities and personnel to collect PNM-PMN under strict resting, supine conditions and thus seated samples are often collected as a necessity. The implications of false-positive PNM-PMN results relate both to clinical care and healthcare costs because these results may lead to extensive downstream investigations for suspected PPGL, especially if the ordering clinician is unfamiliar with the issue of postural variation.

Calgary Lab Services (CLS) provides a high-volume PNM-PMN testing for many referral labs across Canada where samples are drawn without documented attention to patient posture. In addition, CLS receives PNM-PMN samples from subjects seen in the Endocrine Testing Unit (ETU) of the University of Calgary, where patients are carefully prepared for testing and samples drawn according to strict procedural guidelines including supine resting posture. Traditional studies of a diagnostic test typically report sensitivity and specificity which is obviously the preferred statistic when a gold standard comparison is available. However, as that is often unavailable for complex rare diseases in population databases, we have chosen to report a pragmatic surrogate outcome instead, but one that is also immediately relevant to the clinician who must make clinical decisions based upon whether a laboratory result is believed to be true or false. Therefore, the present analysis sought to examine the rates of all abnormal PNM-PMN results derived from a large undifferentiated population being screened for PPGL and to compare the rates of abnormal results with those of a screening population which was sampled under strict sampling conditions. A secondary comparison to matched-method supine PNM-PMN measures from a large international cohort of patients with a confirmed sympathetic PPGL was also undertaken to validate proposed biochemical levels representative of 'high probability for PPGL'.

\section{Methods}

The dataset construction and analysis for the CLS biochemical cohort was reviewed by the University of Calgary Conjoint Health Research Ethics Board and deemed to be exempt from the research ethics review requirement as it is a quality assurance/program evaluation activity; data sharing is not available due to privacy/ ethics restrictions. The international PPGL database was collected and approved for this use according to the regional ethics regulations of each participating center and the local primary investigator. Informed consent was provided by all patients and the full description of this registry has been reported elsewhere (14).

The CLS PNM-PMN dataset includes all results generated between January 2011 and December 2015. CLS processes samples from all Calgary-region health facilities 
in addition to referred-in samples from many Canadian provinces with no stipulations for patient position. An additional set of samples, however, collected from the ETU, was collected under strictly controlled conditions (i.e., following an overnight fast, after resting supine for $30 \mathrm{~min}$ and drawn from a pre-inserted intravenous line) (4). The ETU patients were all those who were sent specifically for resting supine collection by their physicians, mostly endocrinologists and internists. The international PPGL cohort data includes PNM-PMN results of samples collected according to the same strict preparation and posture control in each of the participating centers; the age-adjusted upper reference limit (URL) for PNM was developed at the University of Dresden, Germany and accounts for both age and supine posture. This group of samples also had plasma methoxytyramine levels measured as part of their biochemical profile.

PNM-PMN concentrations are assayed at CLS via an in-house developed liquid chromatography-tandem mass spectrometry (LC-MS/MS) method. Briefly, EDTA plasma specimens undergo sample cleanup and concentration using Oasis HLB solid-phase extraction cartridges. Sample analysis is performed by an Agilent 1100 HPLC system coupled to an Agilent 6410 Mass spectrometer. Separation is performed using a Kinetex 2.6 U HILIC 100A column (Phenomenex; Torrence, CA, USA), with a total run time of $10 \mathrm{~min}$. MS data are acquired in multiple reaction monitoring mode. Calibrators and internal standard material are prepared in-house using stock standards from Cerilliant (Round Rock, TX, USA). QC material is sourced from Recipe (Munich, Germany). The URL used for reporting PNM and PMN are 0.9 and $0.5 \mathrm{nmol} / \mathrm{L}$ (applied to both seated and supine collections) respectively and were adopted from Lagerstedt et al. (15). CLS participates in the Royal College of Pathologists of Australasia Quality Assurance Program (RCPAQAP) for PNM-PMN. Comparison of RCPAQAP sample analysis between CLS and the University of Dresden showed good correlation for both PNM and PMN ( $R^{2}$ was 0.9905 and 0.9764 respectively) and CLS produced results showing an average positive bias of 5\% for both PMN and PNM results across all concentrations. Given the very close agreement between the CLS laboratory and the Dresden laboratory, we are able to adopt the supine, age-adjusted reference range (12) for application to the present data.

Ideally, diagnostic studies should include a 'gold' standard for verification of cases, such as diagnostic imaging and/or surgical pathology. However as a national reference lab, we had no access to clinical and pathological data from outside sites to confirm the diagnosis of PPGL.
Therefore, we used a pragmatic outcome as a 'silver' standard, one that is highly relevant to clinicians: the proportion of patients with PNM-PMN results from the CLS database which were reported above the URL. Given the seriousness of a PPGL diagnosis, no abnormal result for PNM-PMN may be ignored. Thus, the clinician is forced to take a clinical action in response to any abnormal PNMPMN result. For the purposes of this analysis and based on prior published evidence (14), we assumed that any PNMPMN greater than twice the URL would be considered clinically as highly likely diagnostic of PPGL (i.e., a true positive result). Results above the URL but below this twice-normal cut-off were considered indeterminate and possibly comprising both true-positive and false-positive cases; but more likely to represent false-positive results given the rarity of actual PPGL. Importantly, this latter subgroup presents the greatest risk for confusion with a high likelihood of (potentially unnecessary) downstream costs associated with further testing, additional imaging or specialty consultation.

In order to determine the 'abnormal' result rate, the data distribution (PNM and PMN separately) was evaluated according to the URLs reported by CLS (i.e. related to seated upright collections (15)). Stratified analyses were then performed according to age bands (50 years or greater vs less than 50 years). Data from supine-collected ETU samples and international PPGL registry samples were also examined separately. PMN showed no variation by position and so was not considered further in the primary analysis.

Next, in order to discover the potential disagreement in results according to application of different reference ranges by a reporting lab, we analyzed the seated collection population samples using reference ranges established according to patient posture (seated vs supine) and age (12), as well as for the supine ETU samples and international PPGL registry samples separately. Based on these distributions, we determined the proportion of PNM-PMN samples that would be flagged as 'indeterminate but actionable' (i.e. between 1 and $2 x$ the URL) according to different permutations of applied reference ranges, patient posture and age. Finally, we examined the proportion of patients who were detected to have elevated PMN within each predefined category of PNM as an additional marker of possible true PPGL (16).

\section{Statistical analysis}

Descriptive statistics were used to define sample distributions according to rates and proportions; the 
Mann-Whitney $U$ test was used to compare sample population medians of non-normally distributed samples. Chi-square tests were used to compare proportions of categorized populations. A two-tailed $P$ value of $<0.05$ was considered statistically significant. Statistical analysis was performed by GraphPad Prism 6.0 (LaJolla, CA, USA).

\section{Results}

A total of 6138 samples were identified for the study, and these included 5112 outpatient seated collections through CLS (median age 51 years, IQR (36-64), 55\% women), 340 supine samples from the Calgary ETU (median age 50 , IQR (34-62), 56\% women)and 686 samples from the international PPGL database (with each representing an individually confirmed case of PPGL, excluding isolated head and neck paragangliomas) (14).

From the large, unselected, community cohort comprising 5112 samples which were collected while seated, the median PNM was $0.53 \mathrm{nmol} / \mathrm{L}$ (IQR: 0.36-0.79) (Fig. 1A). Individuals who were younger than 50 years of age generally had lower PNM levels compared to those who were older (median $0.46 \mathrm{nmol} / \mathrm{L}$ (IQR: $0.32-67$ ) vs $0.61 \mathrm{nmol} / \mathrm{L}$ (IQR: $0.41-0.88$ ), respectively; $P<0.0001$ ).

The distribution of PNM collected in the Calgary ETU with standardized patient preparation and supine position demonstrated the median PNM was $0.40 \mathrm{nmol} / \mathrm{L}$ (IQR: 0.29-0.58, Fig. 1B). Patients less than 50 years old had a lower median PNM of $0.34 \mathrm{nmol} / \mathrm{L}$ (IQR: 0.27-0.48) compared to individuals 50 years or older, who had a median PNM of $0.47 \mathrm{nmol} / \mathrm{L}$ (IQR: $0.34-0.61 ; P<0.0001$ vs age $<50$ ). The 686 cases of confirmed PPGL were associated with a median PNM of $3.87 \mathrm{nmol} / \mathrm{L}$ (IQR: 1.4-10.1; Fig. 1C). While the vast majority of such subjects, analyzed by seated reference ranges, had PNM values greater than twice the URL, a significant minority (13.1\%) had PNM levels that would be in the normal (seated) reference range if that had been used.

The distributions of PNM results reported according to standard seated reference ranges in comparison to an age-specific, supine URL are shown in Fig. 2 for the 5112 seated community samples from CLS (panel A) and 340 supine collections from the ETU (panel B). In order to examine the distribution of results close to the URL in confirmed PPGL subjects, panel C demonstrates 266 supine samples from the international PPGL database which were close to twice the URL.

The proportion of individuals with concomitantly elevated PMN were examined according to PNM levels
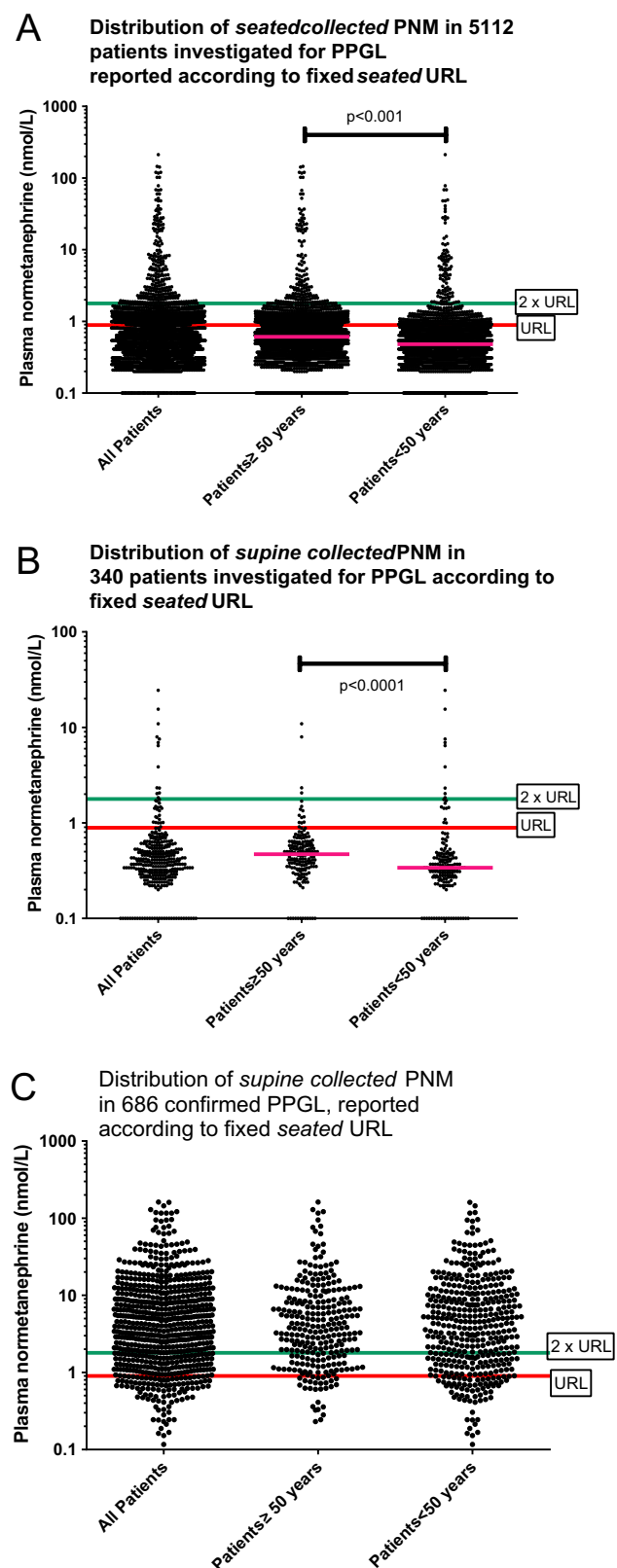

\section{Figure 1}

(A) Median and distribution of PNM in patients suspected of PPGL, referred for seated, outpatient commercial lab sample collection. (B) Distribution of PNM in patients with suspected PPGL referred for resting, supine sample collection. (C) distribution of PNM results in 686 confirmed PPGL cases with samples collected resting, supine. Upper reference limits shown represent commonly used upper reference limits for seated collection samples. PNM, plasma normetanephrine; PPGL, pheochromocytoma/paraganglioma; URL, upper reference limit; $2 \times U R L$, twice the upper reference limit. $P<0.001$ Mann-Whitney $U$ test. A full colour version of this figure is available at https://doi.org/10.1530/EJE-19-0176. 


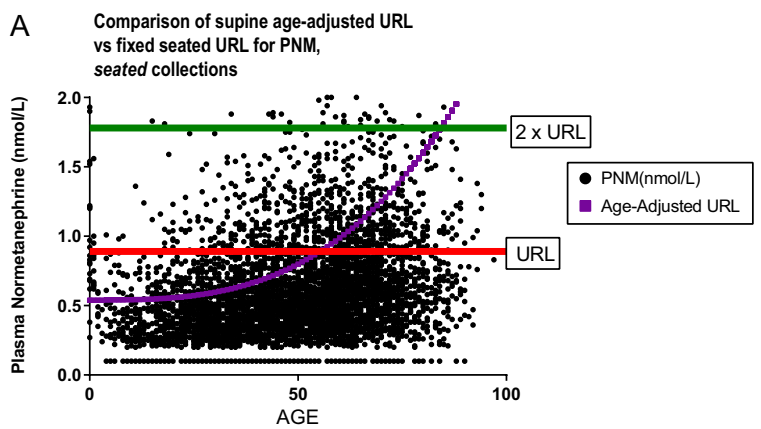

B Comparison of supine age-adjusted URL fixed seated URL for PNM in 340 subjects referred for PPGL investigation,

supine collections

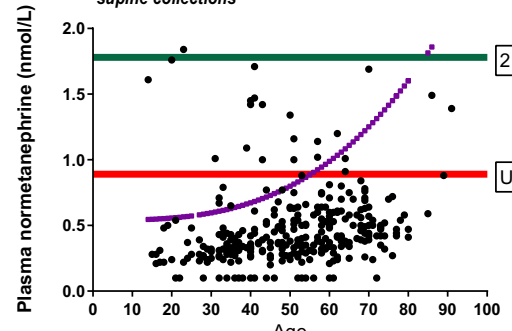

Age

C Comparison of supine age-adjusted URL vs fixed seated URL for PNM in 266 confirmed PPGL patients with fixed seated URL
lower-range PNM, supine collections

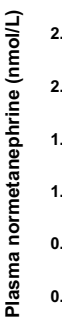
5 *. 1....

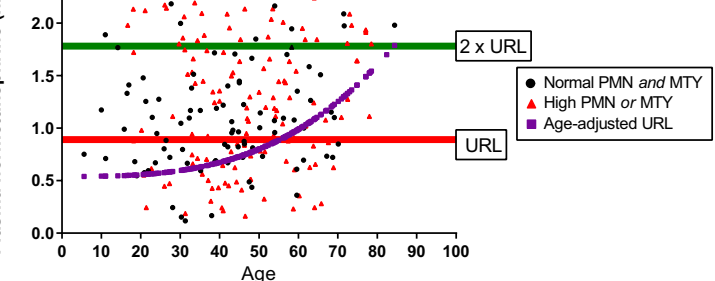

Figure 2

(A) Distribution of seated PNM results in 5112 patients suspected of PPGL according to patient age and with reference to the standard, seated upper reference limit as well as supine, age-adjusted upper reference limit. (B) Distribution of supine, resting PNM in 340 patients suspected of PPGL, according to patient age and with reference to the standard seated upper reference limit as well as supine, age-adjusted upper reference limit. (C) Distribution of supine, resting PNM in patients with confirmed PPGL according to fixed upper reference limits and age adjusted. Note that 462 subjects with much higher PNM are not shown, in order to focus on patients with PNM near the reference limits. Colored triangles in (C) represent patients with normal PNM but high PMN or PMTY. PMN, plasma metanephrine; PMTY, plasma methoxytyramine; PNM, plasma normetanephrine; PPGL, pheochromocytoma/ paraganglioma; URL, upper reference limit. A full colour version of this figure is available at https://doi.org/10.1530/ EJE-19-0176.
(Fig. 3). From community referrals to CLS, whether collected seated or supine, approximately $19-31 \%$ of samples with markedly elevated PNM levels greater than twice URL also had PMN results greater than twice URL and likely represented individuals with true disease (panels A and B). Correspondingly, in patients with confirmed PPGL, 23-31\% had very high PMN independent of PNM (panel C). In contrast, among the undifferentiated samples drawn from the community, normal or minimally elevated PNM were almost never $(<1 \%)$ associated with significant elevations in PMN, suggesting that very few subjects within this subgroup had true PPGL. Nonetheless, almost 99\% of samples with slightly high PNM were not associated with very high PMN, suggesting that most were likely false positives, taken from a general population.

Potential clinical implications are presented for seated collections (Table 1). In total, 19.6\% of PNM results were above the URL, representing a large number of patients for whom additional investigations or consultation would be necessary even though the vast majority would likely be deemed false positives (i.e., given the rare prevalence of PPGL in the population). In patients 50 years or older, PNM levels were generally higher and the proportion of actionable results was also greater (24.9\%). In contrast, the number of results above the URL was much lower when patients had their PNM collected in a supine position (10 vs $19.6 \%, P<0.0001$ ). The rate of slightly positive PNM (between 1 and $2 \times$ URL) was also much lower when patients had their samples collected in a supine compared to seated position ( 6.2 vs $15.0 \%, P<0.0001)$.

Upon restricting the analysis to supine collections and upon applying supine, age-adjusted reference ranges, the proportion of indeterminate PNM results ranged from 5.1 to $5.5 \%$ irrespective of age and represented a marked reduction in slightly elevated PNM (probable false-positive results), especially among those over the age of 50 years (Table 2). Notably, with the addition of age adjustment, markedly elevated PNM levels ( $>2 \times$ URL) were more commonly detected among patients under the age of 50 years $(9.8 \%$ compared to rate of $5.5 \%$ using supine but non-age-adjusted URL), suggesting that the application of the age-adjusted reference range may have yielded higher sensitivity in younger age groups.

\section{Discussion}

PPGL is a very rare disease in an unselected population and therefore, the majority of general patients referred for PPGL screening who have small elevations in PNM 


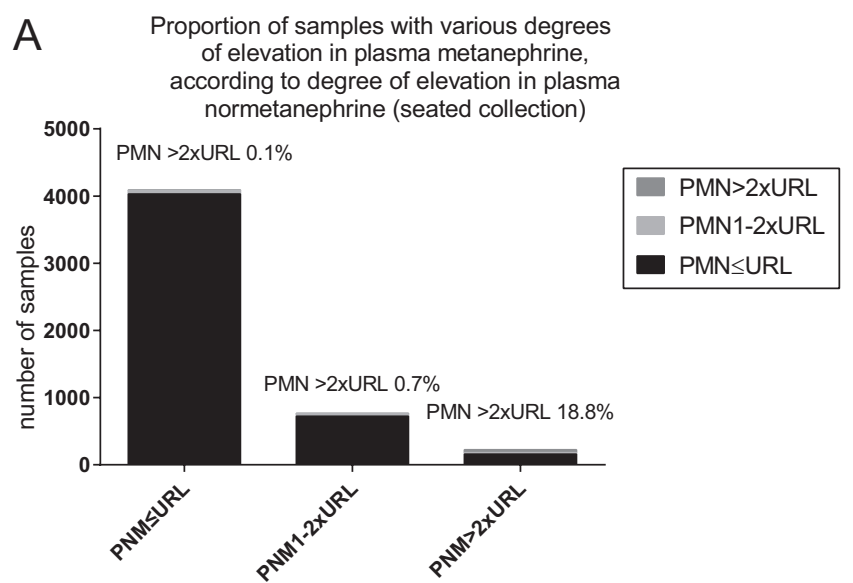

B Proportion of samples with various degrees of elevation in plasma metanephrine, according to degree of elevation in plasma

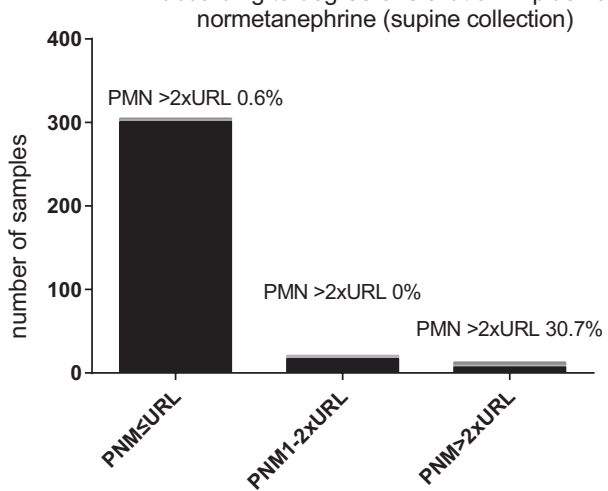

\section{Proportion of PPGL samples with various degrees of elevation in plasma metanephrine, according to degree of elevation in plasma normetanephrine (supine collection)}

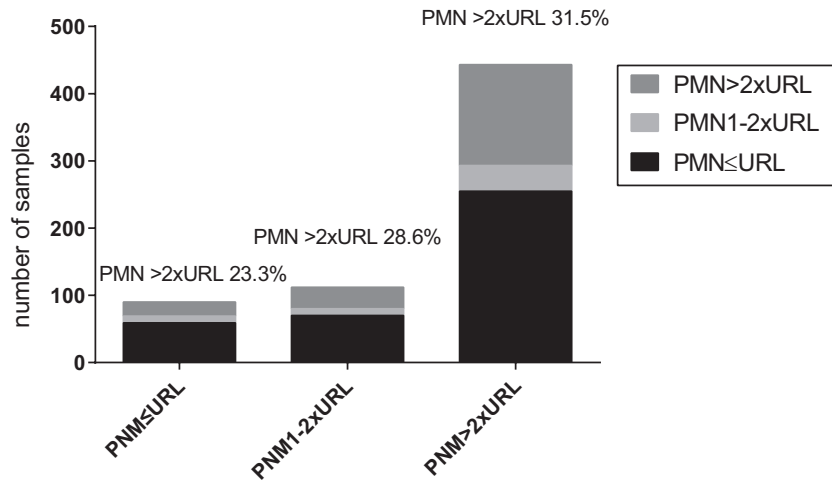

Figure 3

Plasma metanephrine results according to three levels of plasma normetanephrine in a general PPGL screening population (A), a supine endocrine testing population (B) and a cohort of confirmed PPGL (C). PMN, plasma metanephrine; PNM, plasma normetanephrine; PPGL, pheochromocytoma/ paraganglioma, URL, upper reference limit of a (seated) reference range. (less than twice the URL) are very unlikely to have true disease. Nonetheless, elevated PNM results, even those in the indeterminate range, frequently warrant downstream costly investigations and consultations, and may be associated with increased patient anxiety. Extensive adrenal radiologic imaging is also not without risk (17).

Our data confirm prior studies showing a marked difference in PNM results dependent upon posture and support guideline recommendations for supine collection whenever possible (4). A rise in median PNM levels with age is likely under-appreciated when interpreting results in routine clinical care, but our data confirm that patients under the age of 50 years have significantly lower PNM levels than those who are older; failure to account for this difference may result in missed cases among younger individuals. We showed that the potential for misclassification was most evident when reference limits did not account for posture or age. In particular, patients over the age of 50 years were almost four times more likely to have an elevated PNM when collected and reported according to seated vs age-adjusted supine position (24.9 vs $6.8 \%$, respectively). Of these $24.9 \%$, only $1.7 \%$ will be more than $2 \times$ ULN so that the remaining $23.2 \%$ of all such patients will be referred for next-step testing despite being unlikely to have actual PPGL.

However, when patients have PNM collected supine and with supine age-adjusted reference limits used, only $10.8 \%$ of results will be actionable and of those only half will be in the indeterminate range, that is, between 1 and $2 \times$ ULN. Therefore, if laboratories use a policy of seated collection with interpretation using a seated reference range (for which no age-adjusted reference ranges exists), $19.6 \%$ of all subjects referred-in from the general community will have flagged results requiring further investigation. We recognize that supine collection and use of an age-adjusted reference range may be resource intensive and not necessarily feasible for every clinical lab but a policy of referring the 19.6\% flagged high results for such supine testing would be expected to reduce the number of persistent abnormal results requiring investigation by $45 \%$, and the rate of just slightly high (less than twice the URL) to just 5.3\%. Thus, we propose that if PNMs are found to be abnormal from a seated collection, the next step should be a confirmatory re-measurement in a specialized unit that facilitates resting supine collection and offers supine, age-adjusted reference ranges for interpretation. If the usual response to a high PNM level otherwise involves CT/MRI/PET-CT imaging, our proposal would result in a marked reduction in such expensive and potentially harmful imaging 
Table 1 Comparison of 'actionable' CLS results from PNM measures according to posture and age, using fixed, seated upper reference limits $(<0.9 \mathrm{nmol} / \mathrm{L})$.

\begin{tabular}{|c|c|c|c|c|}
\hline Population & Number of patients & $\begin{array}{l}\text { Proportion > fixed URL } \\
\text { (all actionable results) (\%) }\end{array}$ & $\begin{array}{l}\text { Proportion between } \mathbf{1 - 2} \times \text { fixed } \\
\text { URL (actionable but likely FP) (\%) }\end{array}$ & $\begin{array}{l}\text { Proportion }>\mathbf{2} \times \text { fixed URL } \\
\text { (actionable and likely TP) (\%) }\end{array}$ \\
\hline All seated collections & 5112 & 19.6 & $15.0 *$ & 4.6 \\
\hline All seated, age $<50$ & 2398 & 13.4 & 9.6 & 3.8 \\
\hline All seated, age $\geq 50$ & 2714 & 24.9 & 19.7 & 5.3 \\
\hline All supine collections & 340 & 10.0 & $6.2^{*}$ & 3.8 \\
\hline All supine, age $<50$ & 163 & 11.6 & 6.1 & 5.5 \\
\hline All supine, age $\geq 50$ & 177 & 8.5 & 6.3 & 2.2 \\
\hline
\end{tabular}

${ }^{*} P<0.0001$ (chi-square test).

CLS, Calgary Lab Services; FP, false positive; TP, true positive; URL, upper reference limit.

required. Furthermore, this could also reduce the need for traditional clonidine suppression testing or other repeated biochemical measures although some clinicians could still opt for this approach as the next step in those with persistent mild supine PNM elevations with lower pre-test probabilities (18). Common causes of false-positive plasma normetanephrine results such as sleep apnea, anxiety or acute illness should be clinically discerned; the presence of an obvious confounding factor can inform the extent to which additional testing is pursued.

Note that the above suggestions do not apply to PPGL screening in patients at-risk for or with known germline PPGL mutations; in such patients with high pre-test probability, supine age-adjusted testing is mandatory for biochemical screening $(5,11)$. As well, whether seated or supine measures are done, the clinician must be prepared to address pre-test variables such as medications or sleep apnea in order to further reduce false-positive results (19) in all patients.

Comparison of PNM with concomitantly elevated PMN levels may be useful in suspected false-positive cases. As shown in the international PPGL cohort data, at least half of true PPGL patients have marked PMN elevations; very few subjects from an unselected screening population have marked PMN elevations until the PNM is at least twice the URL. Thus, while concomitant elevations in PMN (or plasma methoxytyramine) may increase collective testing specificity, dependence upon the finding of a markedly high paired PMN to identify a likely true positive will only marginally decrease the total proportions of patients needing further investigation for isolated, marginally high PNM results. Of note, elevations in PMN and plasma methoxytyramine should always receive careful attention from the clinician given their specific elevations in patients with adrenal pheochromocytoma (16).

The strength of our analysis rests in the very large sample size ( $>6000$ ) of tests and the fact that such patients necessarily represent the full spectrum of real-life patients who actually undergo PPGL screening. This may be expected to include a wide range of pre-test probabilities in individual patients. Small studies that are enriched in PPGL patients may generate inflated estimates of specificity or positive predictive values from PNM testing; the use of population data and a pragmatic outcome of proportions needing additional assessment speak to the actual experience of clinicians who order these tests. As such, our data shows that a concerning number of patients (almost 20\%) could have inappropriate diagnoses or testing based on PNM measures if the confounding effects of posture and age are not considered in test protocols. Our ability to compare population results to a large international PPGL database is another strength for the study as it validates our assumption that the vast majority of true PPGL have markedly elevated PNM when measured supine. This permits us to conclude that the majority of isolated PNM found to be less than twice the

Table 2 Comparison of 'actionable' results from supine-collected CLS PNM measures according to posture and age, using supine, age-adjusted upper reference limits (see reference (12) for age adjustment explanation).

\begin{tabular}{|c|c|c|c|c|}
\hline Population & $\begin{array}{l}\text { Number of } \\
\text { patients }\end{array}$ & $\begin{array}{c}\text { Proportion > supine age-adjusted URL } \\
\text { (all actionable results) (\%) }\end{array}$ & $\begin{array}{c}\text { Proportion between } 1 \text { and } \mathbf{2} \times \\
\text { age-adjusted URL } \\
\text { (actionable but likely FP) (\%) }\end{array}$ & $\begin{array}{c}\text { Proportion }>\mathbf{2} \times \\
\text { age-adjusted URL } \\
\text { (actionable and likely TP) (\%) }\end{array}$ \\
\hline All supine collections & 340 & 10.8 & 5.3 & 5.6 \\
\hline All supine, age > 50 & 177 & 6.8 & 5.1 & 1.7 \\
\hline All supine, age $<50$ & 163 & 15.3 & 5.5 & 9.8 \\
\hline
\end{tabular}


ULN are indeed going to be false-positive results. Our use of LC-MS/MS measurement methods is another strength of the study, given the known poorer performance of immunoassays for this test (20).

There are several limitations to the present analysis. Our study drew from a laboratory database; lack of clinical data precluded confirmation of clinical diagnoses of PPGL as would have been ideal to report actual test sensitivity and specificity of the tests as conducted. However, this is not intended as a study of sensitivity/ specificity but rather an analysis of potential diagnostic actions that must follow any abnormal PNM test result; given the rarity of PPGL, the vast majority of positive (especially slightly positive) PNM will be false positive and the clinician will want to avoid sending a high number of patients through unnecessary and expensive imaging tests. It is acknowledged that the patients undergoing seated testing in our database were not the same patients getting supine testing and thus comparisons between the two groups cannot be assumed to be exclusively intraindividual postural variation. However, if the patients sent for supine sampling in the ETU represented a population with a higher rate of true PPGL disease, then the difference in proportions of actionable yet likely falsepositive results between seated and supine sampling may be underestimated in the current analysis. As a database study, we were not able to control for the presence of interfering medications or conditions that may have influenced individual results. These represent additional variables that the clinician will need to consider in their clinical interpretation. Lastly, database studies of this nature are unable to inform the clinician regarding the possibility of false negative results; indeed seated sampling and seated reference ranges may lead to small drop in sensitivity, especially among younger patients (6). This highlights the importance of considering the full clinical picture before making a clinical decision on either high or normal PNM results. Ongoing evaluation of PPGL-related test performance through both disease-based registries and laboratory-based databases will be useful to continue to characterize evolving trends in both PPGL presentations and common pitfalls in biochemical diagnosis. Finally, it must be remembered that testing protocols should elicit or at least consider patient experience in local protocol development (21); where feasible, health systems should strive to offer the best test as the initial test and so supine, age-adjusted PNM testing for all samples should be a development goal.

In conclusion, patients from an unselected general care population referred for biochemical PPGL screening may have seated sampling with PNM results reported according to a seated ULN if that is the most feasible for a clinical laboratory. Those with normal results would not undergo further testing unless they had (a) an adrenal mass (especially if younger than 50 years and smaller-size adrenal mass), (b) suspicious lesions for a head/neck or thoracoabdominal PGL, (c) known germline mutation or (d) very high clinical suspicion for PPGL, including prior PPGL; such cases should be referred for supine testing. All PNM results greater than the URL should be referred for supine collection and reported according to a supine age-adjusted reference range prior to further receiving investigations such as imaging or clonidine suppression. Based on our model of this practice, only $5 \%$ of all patients will potentially have imaging and/or suppression testing done for a setting with an indeterminate result between 1 and $2 \times$ URL in supine age-adjusted setting. This should greatly simplify PPGL investigation and avoid the concern and expense otherwise generated by a high false-positive rate of PNM levels.

\section{Declaration of interest}

The authors declare that there is no conflict of interest that could be perceived as prejudicing the impartiality of this study.

\section{Funding}

Alexander Leung is supported by the Hypertension Canada New Investigator Award. This research did not receive any specific grant from any funding agency in the public, commercial or no-for-profit sector. Dr Boyd and Dr Kline conceived the study, collected the data and did the primary analysis. Dr Boyd validated and performed the PNM-MN assays. Dr Leung assisted with the primary analysis. Dr Kline wrote the first draft of the manuscript. Dr Sadrzadeh assisted with the primary data collection and the PNM assay set up. Drs Pamporaki, Pacak, Deutschbein, Fliedner collected the PPGL International cohort data. All authors critically reviewed the manuscript for accuracy and completeness.

\section{Acknowledgements}

The authors gratefully acknowledge the critical review and suggestions provided by Dr Jacques Lenders and Dr Graeme Eisenhofer.

\section{References}

1 Bravo EL \& Tagle R. Pheochromocytoma: state-of-the-art and future prospects. Endocrine Reviews 200324 539-553. (https://doi. org/10.1210/er.2002-0013)

2 Hamidi O, Young Jr WF, Iñiguez-Ariza NM, Kittah NE, Gruber L, Bancos C, Tamhane S \& Bancos I. Malignant pheochromocytoma and paraganglioma: 272 patients over 55 years. Journal of Clinical Endocrinology and Metabolism 2017102 3296-3305. (https://doi. org/10.1210/jc.2017-00992)

3 Clarke B, Ryan G, Fraser J \& Francis L. Uncommon cause of cardiac arrest in the emergency department. Emergency Medicine Australasia 200719 169-172. (https://doi.org/10.1111/j.17426723.2007.00949.x) 
4 Lenders JW, Duh QY, Eisenhofer G, Gimenez-Roqueplo AP, Grebe SK, Murad MH, Naruse M, Pacak K, Young Jr WF \& Endocrine Society. Pheochromocytoma and paraganglioma: an endocrine society clinical practice guideline. Journal of Clinical Endocrinology and Metabolism 201499 1915-1942. (https://doi.org/10.1210/ jc.2014-1498)

5 Eisenhofer G, Prejbisz A, Peitzsch M, Pamporaki C, Masjkur J, Rogowski-Lehmann N, Langton K, Tsourdi E, Pęczkowska M, Fliedner $\mathrm{S}$ et al. Biochemical diagnosis of chromaffin cell tumors in patients at high and low risk of disease: plasma versus urinary free or deconjugated O-methylated catecholamine metabolites. Clinical Chemistry 201864 1646-1656. (https://doi.org/10.1373/ clinchem.2018.291369)

6 Därr R, Pamporaki C, Peitzsch M, Miehle K, Prejbisz A, Peczkowska M, Weismann D, Beuschlein F, Sinnott R, Bornstein SR et al. Biochemical diagnosis of phaeochromocytoma using plasmafree normetanephrine, metanephrine and methoxytyramine: importance of supine sampling under fasting conditions. Clinical Endocrinology 201480 478-486. (https://doi.org/10.1111/cen.12327)

7 Eisenhofer G \& Peitzsch M. Laboratory evaluation of pheochromocytoma and paraganglioma. Clinical Chemistry 201460 1486-1499. (https://doi.org/10.1373/clinchem.2014.224832)

8 Lenders JW, Keiser HR, Goldstein DS, Willemsen JJ, Friberg P, Jacobs MC, Kloppenborg PW, Thien T \& Eisenhofer G. Plasma metanephrines in the diagnosis of pheochromocytoma. Annals of Internal Medicine 1995123 101-109. (https://doi.org/10.7326/00034819-123-2-199507150-00004)

9 Lenders JW, Pacak K, Walther MM, Linehan WM, Mannelli M, Friberg P, Keiser HR, Goldstein DS \& Eisenhofer G. Biochemical diagnosis of pheochromocytoma: which test is best? JAMA $2002 \mathbf{2 8 7}$ 1427-1434. (https://doi.org/10.1001/jama.287.11.1427)

10 Därr R, Kuhn M, Bode C, Bornstein SR, Pacak K, Lenders JWM $\&$ Eisenhofer G. Accuracy of recommended sampling and assay methods for the determination of plasma-free and urinary fractionated metanephrines in the diagnosis of pheochromocytoma and paraganglioma: a systematic review. Endocrine 201756 495-503. (https://doi.org/10.1007/s12020-017-1300-y)

11 Casey R, Griffin TP, Wall D, Dennedy MC, Bell M \& O'Shea PM. Screening for phaeochromocytoma and paraganglioma: impact of using supine reference intervals for plasma metanephrines with samples collected from fasted/seated patients. Annals of Clinical Biochemistry 201754 170-173. (https://doi. org $/ 10.1177 / 0004563216646395)$

12 Eisenhofer G, Lattke P, Herberg M, Siegert G, Qin N, Därr R, Hoyer J, Villringer A, Prejbisz A, Januszewicz A et al. Reference intervals for plasma free metanephrines with an age adjustment for normetanephrine for optimized laboratory testing of phaeochromocytoma. Annals of Clinical Biochemistry 201350 62-69. (https://doi.org/10.1258/acb.2012.012066)

13 Sawka AM, Thabane L, Gafni A, Levine M \& Young WF. Measurement of fractionated plasma metanephrines for exclusion of pheochromocytoma: can specificity be improved by adjustment for age? BMC Endocrine Disorders 20055 1. (https://doi. org/10.1186/1472-6823-5-1)

14 Pamporaki C, Hamplova B, Peitzsch M, Prejbisz A, Beuschlein F, Timmers HJLM, Fassnacht M, Klink B, Lodish M, Stratakis CA et al. Characteristics of pediatric vs adult pheochromocytomas and paragangliomas. Journal of Clinical Endocrinology and Metabolism 2017 102 1122-1132. (https://doi.org/10.1210/jc.2016-3829)

15 Lagerstedt SA, O'Kane DJ \& Singh RJ. Measurement of plasma free metanephrine and normetanephrine by liquid chromatographytandem mass spectrometry for diagnosis of pheochromocytoma. Clinical Chemistry 200450 603-611. (https://doi.org/10.1373/ clinchem.2003.024703)

16 Eisenhofer G, Huynh TT, Hiroi M \& Pacak K. Understanding catecholamine metabolism as a guide to the biochemical diagnosis of pheochromocytoma. Reviews in Endocrine and Metabolic Disorders 20012 297-311. (https://doi.org/10.1023/A:1011572617314)

17 Cawood TJ, Hunt PJ, O'Shea D, Cole D \& Soule S. Recommended evaluation of adrenal incidentalomas is costly, has high false-positive rates and confers a risk of fatal cancer than is similar to the risk of the adrenal lesion becoming malignant; time for a rethink? European Journal of Endocrinology 2009161 513-527. (https://doi.org/10.1530/ EJE-09-0234)

18 Eisenhofer G, Goldstein DS, Walther MM, Friberg P, Lenders JW, Keiser HR \& Pacak K. Biochemical diagnosis of pheochromocytoma: how to distinguish true-from false-positive test results. Journal of Clinical Endocrinology and Metabolism 200388 2656-2666. (https:// doi.org/10.1210/jc.2002-030005)

19 Emms H, Farah G, Shine B, Boot C, Toole B, McFadden M, Lam L, Ou ZQ, Woollard G, Madhavaram H et al. Falsely elevated plasma metanephrine in patients taking midodrine. Annals of Clinical Biochemistry 201855 509-515. (https://doi. org/10.1177/0004563218755817)

20 Weismann D, Peitzsch M, Raida A, Prejbisz A, Gosk M, Riester A, Willenberg HS, Klemm R, Manz G, Deutschbein T et al. Measurements of plasma metanephrines by immunoassay vs liquid chromatography with tandem mass spectrometry for diagnosis of pheochromocytoma. European Journal of Endocrinology 2015172 251-260. (https://doi.org/10.1530/EJE-14-0730)

21 Sussman I, Rothwell T \& Wilson A. Supine or sitting? The voice of the patient stakeholders: economics vs clinically and medically sound. Clinical Endocrinology 201582 464-465. (https://doi. org/10.1111/cen.12624)

Received 10 April 2019

Revised version received 26 June 2019

Accepted 10 July 2019 